\title{
Bridging the Microalga Mesoscale: High-throughput Systems Biology and Bioimaging for Guided Biodesign of Microalgae
}

\author{
Chuck Smallwood $^{1}$, Jian-Hua Chen ${ }^{2}$, James Evans ${ }^{3}$ and Gerry McDermott ${ }^{4}$ \\ ${ }^{1}$ Sandia National Laboratory, Albuquerque, New Mexico, United States, ${ }^{2}$ Lawrence Berkeley National \\ Laboratory and UCSF, Berkeley, California, United States, ${ }^{3}$ Pacific Northwest National Laboratory, \\ Richland, Washington, United States, ${ }^{4}$ University of California, San Francisco, San Francisco, California, \\ United States
}

The intracellular space harbors an array of highly dynamic molecular mechanisms and structural changes that interact on the mesoscale and dictate overall phenotype. The precise location of lipid biosynthesis and accumulation in phototrophic microalga systems remains unclear. We utilized an array of interdisciplinary approaches such as structural biology, multi-omics, and multi-scale microscopy to probe subcellular ultrastructure, composition, and molecular interactions to understand the complex connections occurring within microalgae cells during nitrogen and carbon stress. These integrated analyses reveal the formation of lipids under various nitrogen and carbon components within the smallest eukaryote Ostreococcus tauri using label-free and label-based bioimaging, proteomics, lipidomics, and soft x-ray nanotomography. These multimodal data provided a holistic view into the metabolic mechanisms and structural reorganization of $O$. tauri that enabled targeted biodesign efforts.

Ostreococcus tauri is an ancient phototrophic microalga that possesses favorable genetic and cellular characteristics for reductionist studies probing biosystem design and dynamics. Here multimodal bioimaging and multi-omics techniques were combined to interrogate $\mathrm{O}$. tauri cellular changes in response to variations in bioavailable nitrogen and carbon ratios. Confocal microscopy, stimulated Raman scattering, and cryo-soft x-ray tomography revealed whole cell ultrastructural dynamics and composition while proteomic and lipidomic profiling captured changes at the molecular and macromolecular scale.

Despite several energy dense long-chain triacylglycerol lipids showing more than 40-fold higher abundance under $\mathrm{N}$ deprivation, general protein abundances directly associated with lipid biogenesis showed only a few increases in protein expression. However, the entire pathway for starch granule biosynthesis was highly upregulated during nitrogen deprivation. These results suggest that despite visualization of neutral lipid increases, when elevated bicarbonate is introduced cellular energy is expended on increasing protein abundance preferentially for starch over lipid biosynthesis pathways. Thus, post-translational control of lipid chain formation is the likely actor responsible for increases in cellular lipid content. Additionally, three of the five most downregulated and five of the ten most upregulated proteins during severe nitrogen depletion were unnamed protein products that warrant additional biochemical analysis and functional annotation to control carbon transformation dynamics in this smallest eukaryote. Here, an integrated analysis of bioimaging, proteomic, and lipidomic characterization was used to investigate $O$. tauri cellular response to varying C: $\mathrm{N}$ ratios. Our results provide additional understanding of $\mathrm{C}$ storage and energy transformation pathways within the microalgae $O$. tauri and identify new proteins to target for future bioengineering efforts in this organism and other tractable algae strains. 


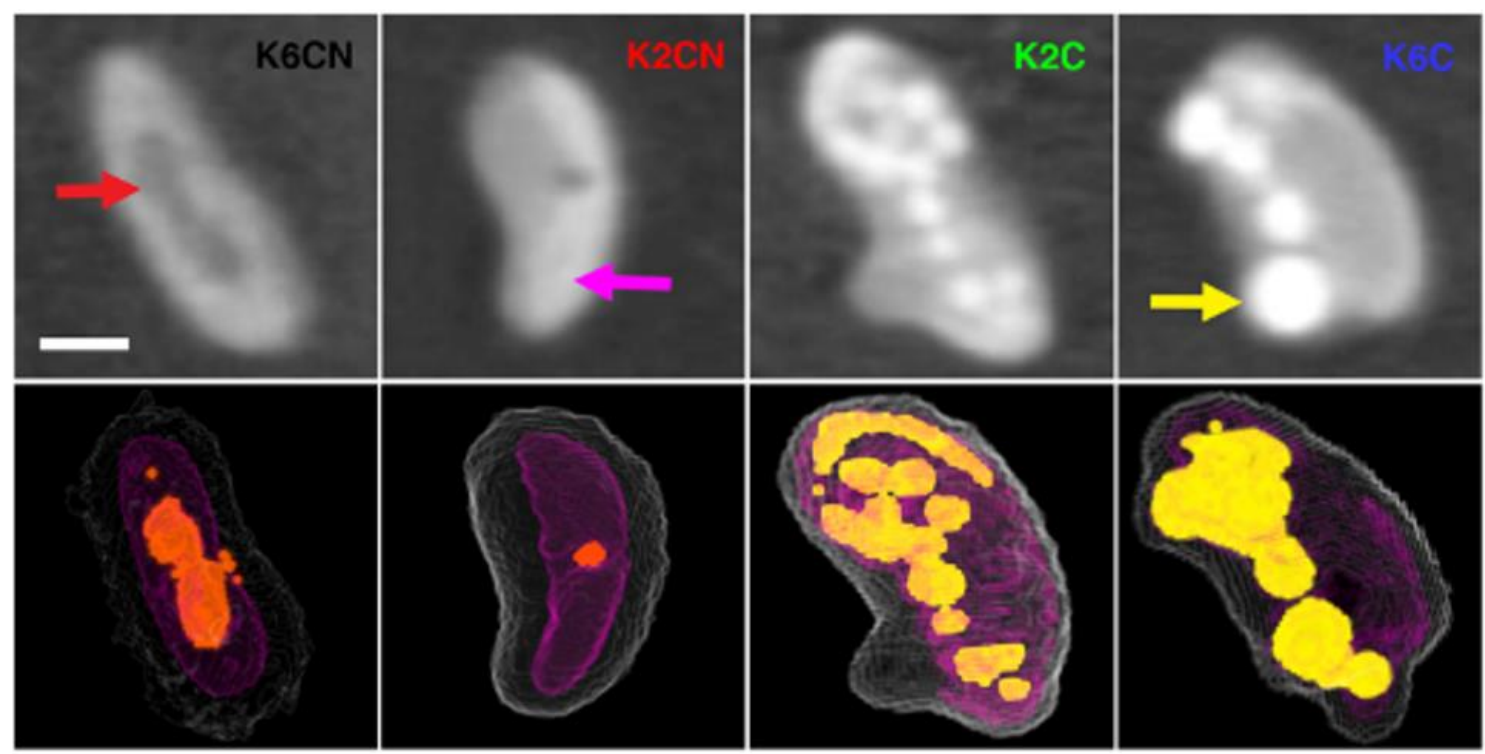

Figure 1. Label-free cryogenic soft x-ray nanotomography images of cells that were cryogenically frozen in microcapillaries and imaged after 72 hours. 


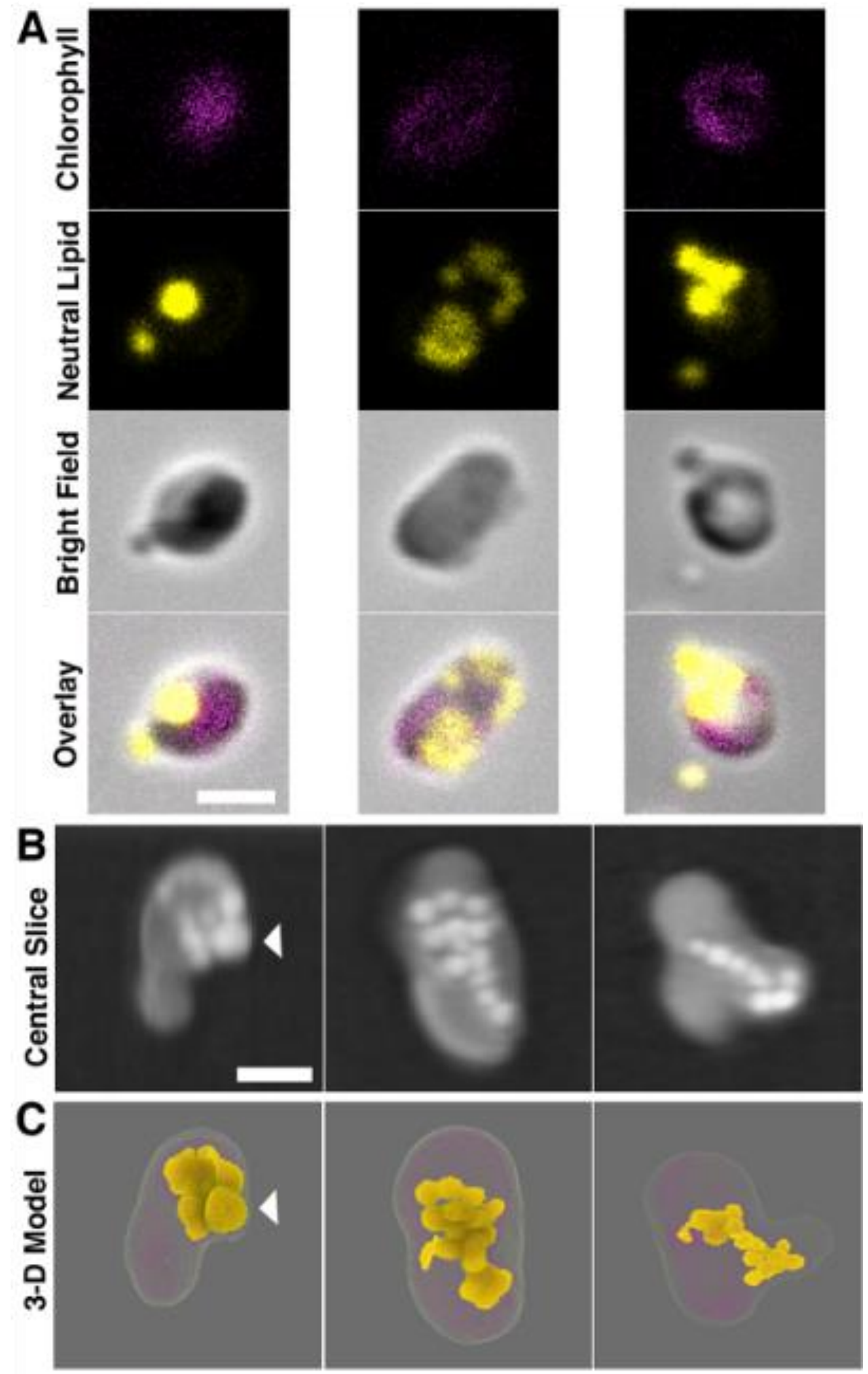


Figure 2. Combining our microscopy modalities enabled capture of different distributions of lipid bodies. We found cells in both optical microscopy experiments and SXT experiments with similar lipid distributions which indicated unique mechanisms of intracellular lipid accumulation and distribution. We also found curious structures in the same cultures for both modalities that will require further exploration, characterization, and possibly classification.

\section{References}

- Smallwood, C.R., Chrisler, W., Chen J., Patello E., Boudreau R., McDermott G., Le Gros M., Evans J.E., Ostreococcus tauri is a high-lipid content green algae that extrudes clustered lipid droplets. 2018 bioRXiv (under review in Scientific Reports) DOI: https://doi.org/10.1101/249052

- Smallwood, C.R., Hill, E.A., Chrisler, W. B., Brookreson, J.T., and Evans, J.E., Optimizing bioreactor growth of the smallest eukaryote 2018 bioRXiv DOI: https://doi.org/10.1101/291211 (under review in Algal Research)

- Smallwood, C.R., Chen, J., Kyle, J.E., Chrisler, W. B., Hixson, K.K., Nicora, C.D., Moore, R.J., Purvine, S.O. and Evans, J.E., Integrated systems biology and imaging of the smallest free-living eukaryote Ostreococcus tauri. 2018 bioRXiv DOI: https://doi.org/10.1101/293704 (submitted to mSystems) 